University of Nebraska - Lincoln

DigitalCommons@University of Nebraska - Lincoln

Papers in Natural Resources

Natural Resources, School of

2009

\title{
Analytical solution of the coupled 2-D turbulent heat and vapor transport equations and the complementary relationship of evaporation
}

Jozsef Szilagyi

University of Nebraska-Lincoln, jszilagyi1@unl.edu

Janos Jozsa

Budapest University of Technology and Economics

Follow this and additional works at: https://digitalcommons.unl.edu/natrespapers

Part of the Natural Resources and Conservation Commons, Natural Resources Management and Policy Commons, and the Other Environmental Sciences Commons

Szilagyi, Jozsef and Jozsa, Janos, "Analytical solution of the coupled 2-D turbulent heat and vapor transport equations and the complementary relationship of evaporation" (2009). Papers in Natural Resources. 906.

https://digitalcommons.unl.edu/natrespapers/906

This Article is brought to you for free and open access by the Natural Resources, School of at DigitalCommons@University of Nebraska - Lincoln. It has been accepted for inclusion in Papers in Natural Resources by an authorized administrator of DigitalCommons@University of Nebraska - Lincoln. 


\title{
Analytical solution of the coupled 2-D turbulent heat and vapor transport equations and the complementary relationship of evaporation
}

\author{
Jozsef Szilagyi ${ }^{\mathrm{a}, \mathrm{b}, *}$, Janos Jozsa ${ }^{\mathrm{a}}$ \\ a Department of Hydraulic and Water Resources Engineering, Budapest University of Technology and Economics, H-1111 Muegyetem rkp. 3-9, Budapest, Hungary
}

${ }^{\mathrm{b}}$ School of Natural Resources, University of Nebraska-Lincoln, Conservation and Survey Division, 113 Nebraska Hall, Lincoln, NE 68583, USA

\section{A R T I C L E I N F O}

\section{Article history:}

Received 30 April 2008

Received in revised form 26 March 2009

Accepted 30 March 2009

This manuscript was handled by $\mathrm{K}$. Georgakakos, Editor-in-Chief, with the assistance of Anna P. Barros, Associate Editor

\section{Keywords:}

Complementary relationship of evaporation Coupled turbulent heat and moisture

transport equations

Evaporation

Evapotranspiration

\section{S U M M A R Y}

The existence of a symmetrical complementary relationship (CR) in evaporation has been hypothesized in the hydrologic literature but the conditions required have not been investigated in much detail. In this study it is shown that under near-neutral atmospheric conditions and a constant energy term at the evaporating surface, the analytical solution of the coupled turbulent diffusion equations of heat and vapor transport across a moisture discontinuity of the surface yields a symmetrical CR between the evaporation rate of the uniform drying land upwind of the discontinuity and the mean evaporation rate of the wet area provided, the latter has a proper fetch (i.e., along-the-wind extent). This fetch is a function of the air stability parameter and the assumed uniform surface roughness value, and it is in the order of a $100 \mathrm{~m}$ for a smooth surface under near-neutral atmospheric conditions. The analytically derived mean evaporation rates of such a smooth wet surface compare well, i.e., within $10 \%$, to the Penman equation estimates, most frequently employed within the CR framework.

(c) 2009 Elsevier B.V. All rights reserved.

\section{Introduction}

The complementary relationship (CR) of evaporation, first introduced by Bouchet (1963), is an important tool for the practicing hydrologist because it can estimate actual evaporation $(E)$ from climatic variables that are regularly observed at standard meteorological stations. This is unique because other existing evaporation estimation methods require measurements (a) obtained at two different heights above the ground (Bowen-ratio instruments); (b) taken by fast response sensors (eddy-covariance stations), or (c) by remote sensing platforms. Still others need information of the vegetation status of the land (Penman-Monteith equation (Monteith, 1973)) or depend on the choice of the soil-moisture tracking algorithm to maintain a book keeping of the incoming and outgoing water fluxes of the area in question within the confines of a lumped or distributed hydrologic model.

A recent upsurge in CR-based evaporation studies (Hobbins et al., 2001a,b; Szilagyi, 2001a, 2007; Szilagyi et al., 2001; Crago

\footnotetext{
* Corresponding author. Address: School of Natural Resources, University of Nebraska-Lincoln, Conservation and Survey Division, 113 Nebraska Hall, Lincoln, NE 68583, USA.

E-mail address: jszilagyi1@unl.edu (J. Szilagyi).
}

and Crowley, 2005; Ramirez et al., 2005; Kahler and Brutsaert, 2006; Pettijohn and Salvucci, 2006; Szilagyi and Jozsa, 2008, 2009) combined with a controversy about the existence of a true complementary relationship between potential $\left(E_{p}\right)$ and actual evaporation rates (LeDrew, 1979; McNaughton and Spriggs, 1989; Kim and Entekhabi, 1997; Lhomme, 1997a,b; Sugita et al., 2001; Szilagyi, 2001b; Lhomme and Guilioni, 2006) motivated this study. It is aimed to look at how the increase in sensible heat flux over a drying land surface actually becomes transformed into a corresponding increase in potential evaporation rates, especially when this transformation is not uniform in space.

It is important to mention that any theory requires certain predefined conditions to exist under which it is supposed to be valid. In reality, such exact conditions may not always be found or just rarely. Here an attempt is made to show that a true complementarity indeed exists between actual $(E)$ and potential evaporation $\left(E_{p}\right)$ rates provided certain conditions, discussed in detail below, are maintained. This work however does not try to investigate how the symmetry breaks down and the CR becomes modified when the required conditions are not met in reality. These issues are dealt with in, e.g., Brutsaert and Parlange (1998), Kahler and Brutsaert (2006), Szilagyi (2007), and Szilagyi and Jozsa (2008).

The CR (Bouchet, 1963) can be expressed by the simple equation 
$E+E_{p}=\eta E_{w}$

where $E_{w}$ is the so-called wet environment evaporation rate which would result from a uniform wet surface of regional extent. The proportionality coefficient, $\eta$, can be a constant (Brutsaert and Stricker, 1979; Kahler and Brutsaert, 2006) or a temperature-dependent variable (Szilagyi, 2007). When $\eta=2$, the relationship is symmetric around an assumed constant $E_{w}$ value resulting from a similarly constant energy rate $\left(Q_{n}\right)$ at the surface (fully consumed by sensible $[H]$ and latent heat $[L E]$ fluxes between the land and the air), meaning that $E$ increases/decreases by the same amount as $E_{p}$ decreases/increases. Note that the term, potential evaporation, $E_{p}$, in essence is designed to specify the evaporation rate that would take place from a large area under the same atmospheric conditions $E$ is observed, were moisture not a limiting factor. Following Brutsaert and Parlange (1998), Szilagyi (2007) and Szilagyi and Jozsa (2008) concluded that as long as there is no heat transfer from a warmer drying land to a cooler wet surface (or when this heat transfer is negligible due to the size of the areas involved) across the solid boundary of the freely evaporating surface, (1) can become symmetric under time-invariant $Q_{n}$, wind and turbulent diffusion coefficient conditions. To further check this largely speculative claim, the coupled 2-D turbulent heat and vapor transport equations will be utilized after Yeh and Brutsaert (1971) and Brutsaert (1982) in this study.

\section{Analytical solution of the coupled turbulent heat and vapor transport equations}

Let's consider a sudden moisture and temperature discontinuity at the land surface spreading to infinity perpendicular to the prevailing mean wind, $\bar{u}$, blowing along the $x$-axis of a Cartesian coordinate system. Since everything is assumed to be homogeneous along the $y$-axis, the other two components of the mean wind vector can be considered zero without loss of generality. Let's denote by $K_{v}$ and $K_{h}$ the vertical tensor components of the turbulent diffusivity for vapor and heat, and assume that they are comparable, i.e., $K_{v} \approx K_{h}=K$. By applying a first-order closure approach (i.e., the $K$ theory) for the turbulent fluxes the steady vapor and heat transport equations become

$\bar{u} \frac{\partial \bar{q}}{\partial x}=\frac{\partial}{\partial z}\left(K \frac{\partial \bar{q}}{\partial z}\right)$

$\bar{u} \frac{\partial \bar{T}}{\partial x}=\frac{\partial}{\partial z}\left(K \frac{\partial \bar{T}}{\partial z}\right)$

where $\bar{q}$ is the mean specific humidity, $\bar{T}$ is the mean air temperature, a good substitute for the required mean potential temperature due to the close proximity to the land surface. The surface roughness is assumed to be uniform, while the prescribed equilibrium $\bar{u}(z)=a z^{m}$ and $K(z)=b z^{n}$ profiles are further assumed to remain unchanged across the discontinuity. From experimental data $a=(5.5 /$ $7 m) u_{*}\left(z_{0}\right)^{-m}, b=u_{*} z_{0}^{m} /(5.5 m)$ and $n=1-m$ can be written, where $u_{*}$ is the friction velocity and $z_{0}$ the roughness height of the surface, but they are not needed to be specified this way for the solution of (2).

Upwind of the discontinuity (from here on the subscript ' $a$ ' will refer to the non-wet 'arid' conditions) let the specific humidity, $\bar{q}_{a}(z)$, and temperature, $\bar{T}_{a}(z)$, profiles be in an equilibrium, meaning

$\frac{\partial}{\partial z}\left(K \frac{\partial \bar{T}_{a}}{\partial z}\right)=\frac{\partial}{\partial z}\left(K \frac{\partial \bar{q}_{a}}{\partial z}\right)=0$.

At the wet surface, i.e., $0<x<x_{f}$, the specific humidity is a function of its temperature, although this is not a necessary assumption for the solution. Here $x_{f}$ is the extent (or fetch) of the wet surface along the $x$-axis. Let also the net energy flux be zero at the surface, upand downwind of the discontinuity, while let the incoming radiation, $R_{d a}$ and $R_{d}$, be constant, however not necessarily the same due to possible differences in the albedo as a result of the moisture contrast. Note, the thermal radiation of the surface, treated as a grey body with emissivity, $\varepsilon$, will not be constant in general as it maybe a function of the surface temperature due to changes in the moisture content of the land surface. Let's assume here that heat conduction at the surface, $G_{a}$ and $G$ into the soil is constant, although they may depend on the surface temperature, as discussed by Yeh and Brutsaert (1971) who also formulated a solution of (2) for this latter, more general case.

Let the boundary conditions (BC) be formulated first for the 'arid' surface

$$
\begin{aligned}
& \bar{q}_{a}=q_{a s}, \quad \bar{T}_{a}=T_{a s} \quad \text { at } z=0 \\
& -c_{p} \rho K \frac{\partial \bar{T}_{a}}{\partial z}-L_{e} \rho K \frac{\partial \bar{q}_{a}}{\partial z}+\varepsilon \sigma T_{a s}^{4}+G_{a}=R_{d a} \quad \text { at } z=0 \\
& -c_{p} \rho K \frac{\partial \bar{T}_{a}}{\partial z}=H_{a}, \quad-\rho K \frac{\partial \bar{q}_{a}}{\partial z}=E_{a}, \quad \text { at } z=0
\end{aligned}
$$

and now for the wet surface

$$
\begin{array}{ll}
\bar{q}=\bar{q}_{a}(z), \quad \bar{T}=\bar{T}_{a}(z) & \text { at } x=0, z>0 \\
\bar{q}=q_{s}\left(T_{s}\right) & \text { at } 0<x<x_{f}, z=0 \\
-c_{p} \rho K \frac{\partial \bar{T}}{\partial z}-L_{e} \rho K \frac{\partial \bar{q}}{\partial z}+\varepsilon \sigma T_{s}^{4}+G=R_{d} & \text { at } 0<x<x_{f}, z=0 \\
-c_{p} \rho K \frac{\partial \bar{T}}{\partial z}=H_{a}, \quad-\rho K \frac{\partial \bar{q}}{\partial z}=E_{a} & \text { at } x>x_{f}, z=0
\end{array}
$$

where $c_{p}$ is the specific heat of air at constant pressure, $L_{e}$ the latent heat of vaporization of water, $\sigma$ the Stefan-Boltzmann constant, $\rho$ the air density, $H_{a}$ and $E_{a}$ the heat and water vapor fluxes from the land surface up- and downwind of the wet surface. Note that only either $T_{a s}$ or $q_{a s}$ can be arbitrary, since for a given $R_{d a}$ and $G_{a}$ they together must satisfy the second BC of the arid surface above.

Eq. (2) with the specified equilibrium profiles and BCs was first solved analytically by Laikhtman (1964) and later for the more general soil heat conduction case by Yeh and Brutsaert (1971). Let the following terms be defined as

$$
\begin{aligned}
& c_{1}=c_{p} \rho b\left(T_{m}-T_{a s}\right)\left(\frac{a}{b x_{f}}\right)^{v}(1-n)^{1-2 v} \\
& c_{2}=4 \varepsilon \sigma T_{a s}^{3}\left(T_{m}-T_{a s}\right) \\
& c_{3}=L_{e} \rho b\left(q_{m}-q_{a s}\right)\left(\frac{a}{b x_{f}}\right)^{v}(1-n)^{1-2 v} \\
& c_{4}=\left(R_{d}-G\right)-\left(R_{d a}-G_{a}\right) \\
& c_{5}=\left.\frac{T_{m}-T_{a s}}{q_{m}-q_{a s}} \frac{d q^{*}}{d T}\right|_{T=\langle T\rangle}=\frac{T_{m}-T_{a s}}{q_{m}-q_{a s}} \alpha_{q} \\
& c_{6}=\frac{q_{a s}^{*}-q_{a s}}{q_{m}-q_{a s}} \\
& \omega=\frac{c_{2} v^{1-2 v} \Gamma(v)}{\left(c_{1}+c_{3} c_{5}\right) \Gamma(1-v)}
\end{aligned}
$$

where $T_{m}$ and $q_{m}$ are some representative temperature and specific humidity of the wet surface, $v=(1-n) /(2+m-n)$, $\Gamma$ is the complete gamma function, and $q^{*}$ is a value at the saturated specific humidity curve, the slope of which, $\alpha_{q}$, is to be taken at temperature, $\langle T\rangle$. Yeh and Brutsaert (1971) specified $\langle T\rangle$ as $T_{a s}$, but applying a temperature $\langle T\rangle=\left(T_{a s}+T_{m}\right) / 2$ in the numerical evaluation of the analytical solution yields significantly improved accuracy as discussed later. With the above terms the water vapor and heat flux from the wet surface can be obtained as 


$$
\begin{aligned}
E= & -\left.\rho K \frac{\partial \bar{q}}{\partial z}\right|_{z=0}=E_{a}+c_{p} \rho b\left(\frac{a}{b x_{f}}\right)^{v}(1-n)^{1-2 v} \\
& \times \frac{q_{a s}^{*}-q_{a s}}{\Gamma(v) v^{1-2 v}\left(c_{p}+\alpha_{q} L_{e}\right)} \xi^{-v} \\
& +\frac{\alpha_{q}\left[c_{4}+c_{2} c_{3} c_{6} /\left(c_{1}+c_{3} c_{5}\right)\right]}{c_{p}+\alpha_{q} L_{e}} \sum_{i=0}^{\infty} \frac{(-\omega)^{i} \xi^{i v}}{\Gamma(1+i v)} \\
H= & -\left.c_{p} \rho K \frac{\partial \bar{T}}{\partial z}\right|_{z=0}=H_{a}-c_{p} \rho b\left(\frac{a}{b x_{f}}\right)^{v}(1-n)^{1-2 v} \\
& \times \frac{L_{e}\left(q_{a s}^{*}-q_{a s}\right)}{\Gamma(v) v^{1-2 v}\left(c_{p}+\alpha_{q} L_{e}\right)} \xi^{-v}+\frac{c_{p}\left[c_{4}+c_{2} c_{3} c_{6} /\left(c_{1}+c_{3} c_{5}\right)\right]}{c_{p}+\alpha_{q} L_{e}} \\
& \times \sum_{i=0}^{\infty} \frac{(-\omega)^{i} \xi^{i v}}{\Gamma(1+i v)}
\end{aligned}
$$

where $\xi=x / x_{f}$. The temperature and specific humidity of the wet surface results as

$$
\begin{aligned}
T_{s}= & T_{a s}-\frac{L_{e}\left(q_{a s}^{*}-q_{a s}\right)}{c_{p}+\alpha_{q} L_{e}}+\frac{v^{1-2 v} \Gamma(v)\left[c_{4}+c_{2} c_{3} c_{6} /\left(c_{1}+c_{3} c_{5}\right)\right]}{\Gamma(1-v) \rho b\left[a /\left(b x_{f}\right)\right]^{v}(1-n)^{1-2 v}\left(c_{p}+\alpha_{q} L_{e}\right)} \\
& \times \sum_{i=0}^{\infty} \frac{(-\omega)^{i} \xi^{v+i v}}{\Gamma(1+v+i v)} \\
q_{s}= & q_{a s}+\frac{c_{p}\left(q_{a s}^{*}-q_{a s}\right)}{c_{p}+\alpha_{q} L_{e}}+\frac{\alpha_{q} v^{1-2 v} \Gamma(v)\left[c_{4}+c_{2} c_{3} c_{6} /\left(c_{1}+c_{3} c_{5}\right)\right]}{\Gamma(1-v) \rho b\left[a /\left(b x_{f}\right)\right]^{v}(1-n)^{1-2 v}\left(c_{p}+\alpha_{q} L_{e}\right)} \\
& \times \sum_{i=0}^{\infty} \frac{(-\omega)^{i} \xi^{v+i v}}{\Gamma(1+v+i v)}
\end{aligned}
$$

For the full solution describing $\bar{T}(x, z)$ and $\bar{q}(x, z)$ over the wet surface, see Laikhtman (1964) or Yeh and Brutsaert (1971).

By assuming that at the surface the available energy, $Q_{n}$, rather than $R_{d}$ or $R_{d a}$, remains constant in time as the originally uniformly wet area dries out for $x \leqslant 0$ (and also for $x>x_{f}$ ), i.e., $Q_{n}=R_{d a}-G_{a}-\varepsilon \sigma T_{a s}^{4}=R_{d}-G-\varepsilon \sigma T_{s}^{4}=$ const., then the thermal radiation and soil heat conduction terms drop out from the BCs (since this way they never get defined), and so do the $c_{2}$ and $c_{4}$ terms as well above, the latter because both $R_{d a}$ and $R_{d}$ become replaced by the same constant $Q_{n}$. As a consequence, the third terms of the right-hand-side (r.h.s.) of (4)-(7) vanish too, yielding

$$
\begin{aligned}
E= & -\left.\rho K \frac{\partial \bar{q}}{\partial z}\right|_{z=0}=E_{a}+c_{p} \rho b\left(\frac{a}{b x_{f}}\right)^{v}(1-n)^{1-2 v} \\
& \times \frac{q_{a s}^{*}-q_{a s}}{\Gamma(v) v^{1-2 v}\left(c_{p}+\alpha_{q} L_{e}\right)} \xi^{-v} \\
H= & -\left.c_{p} \rho K \frac{\partial \bar{T}}{\partial z}\right|_{z=0}=H_{a}-c_{p} \rho b\left(\frac{a}{b x_{f}}\right)^{v}(1-n)^{1-2 v} \\
& \times \frac{L_{e}\left(q_{a s}^{*}-q_{a s}\right)}{\Gamma(v) v^{1-2 v}\left(c_{p}+\alpha_{q} L_{e}\right)} \xi^{-v} \\
T_{s}= & T_{a s}-\frac{L_{e}\left(q_{a s}^{*}-q_{a s}\right)}{c_{p}+\alpha_{q} L_{e}} \\
q_{s}= & q_{a s}+\frac{c_{p}\left(q_{a s}^{*}-q_{a s}\right)}{c_{p}+\alpha_{q} L_{e}}
\end{aligned}
$$

From (8) and (9) now it becomes clear that the sensible, $H$, and latent heat fluxes, $L E$, change at the same rate along the wet surface, but opposite in sign, since (8) multiplied by $L_{e}$ yields the same second term on the r.h.s. as (9). From (10) it furthermore follows that the temperature of the wet surface is constant along the fetch. A constant wet surface temperature along the wet patch means that any point of the wet surface, even if it is located infinitely far along the patch, should have the same temperature response to an upwind aridity change of the environment. However, along the wet fetch the advected drier and warmer air blends more and more into the wet environment by becoming increasingly more humid and cooler, which means that after a certain distance it will have no effect on the temperature of the wet surface, therefore a spatially constant temperature of the wet patch can only be maintained if the wet surface temperature remains constant in time as well. This conclusion of the wet surface temperature invariance to aridity changes under a constant $Q_{n}$ (and unchanged $\bar{u}(z)$ and $K(z)$ profiles) has already been drawn speculatively by Morton (1983) and more recently by Szilagyi (2001b) and Szilagyi and Jozsa (2008). While Yeh and Brutsaert (1971) also discussed this possibility of a balance in the sensible and latent heat fluxes along the wet surface they did not explicitly specify when could this be expected, i.e., when $Q_{n}$ is constant.

Dividing (10) by (11), the following equation results

$\frac{T_{s}-T_{a s}}{q_{s}-q_{a s}}=-\frac{L_{e}}{c_{p}}=\frac{\Delta T_{a s}}{\Delta q_{a s}}$

which is similar to the wet-bulb temperature equation written now for the drying land surface. Here, $\Delta$ designates a temporal change. The equation at the l.h.s. of (12) has been published by Yeh and Brutsaert (1971), but not the r.h.s. which follows from a constant wet surface temperature realization under a constant $Q_{n}$, provided the area was uniformly wet initially. Eq. (12) can help with actual evaporation estimation when the land surface temperature is monitored by satellite-mounted remote sensing instruments (Szilagyi and Jozsa, 2009), since it relates the surface temperature increase of the land to a change in its moisture status under a constant $Q_{n}$. With additional air temperature and humidity measurements sensible and latent heat fluxes can thus be derived (Szilagyi and Jozsa, 2009).

In (12) for any constant $T_{s}$ either $T_{a s}$ or $q_{a s}$ can be specified and the other calculated. However, when the so-derived pair of $T_{a s}$ and $q_{a s}$ values is substituted back into (10) and (11) with $\alpha_{q}$ evaluated at $T_{a s}$ as defined by Yeh and Brutsaert (1971), a large difference in the $T_{s}$ values between (10) and (12) can be observed (Table 1) whenever the $T_{a s}-T_{s}$ or $q_{a s}-q_{s}$ difference itself is large. This is so because the derivation of the analytical solution of $(2)$ is based on a linearization that requires the temperature (and humidity) change at the drying surface to be small. The discrepancy can however be significantly improved if $\alpha_{q}$ is evaluated at $\langle T\rangle=\left(T_{a s}+T_{m}\right) / 2$ rather, as has been mentioned before (Table 1 ). This temperature replacement however is expected to only slightly affect the numerical values of the analytical solution for $H$ and $E$.

Fig. 1 displays the specific humidity and air temperature distributions around a wet surface having a fetch of $50 \mathrm{~m}$ in the surrounding drying land derived by numerical integration of (2) in the finite-element model, FLEXPDE (http://www.pdesolutions.com). Naturally the same could have been achieved by the analytical solution from Laikhtman (1964) or Yeh and Brutsaert (1971) for the $\bar{q}$ and $\bar{T}$ profiles over the wet surface and the below derived equilibrium profiles for the drying land. With the prescribed $q_{a s}$ value, $T_{a s}$ was obtained from (12). Initially the uniformly wet surface was assumed to have a surface temperature of $20^{\circ} \mathrm{C}$ which was to be conserved over the wet surface due to the application of (12). Note the reversal of the temperature profile around the leading edge of the wet area as the hotter air runs over the cooler surface, supplying heat toward the wet surface to be fully consumed by the corresponding increase in evaporation.

From the second terms of the r.h.s. of (8) and (9) it is obvious that neither the evaporation nor the sensible heat flux is constant along the wet surface (Fig. 2), even when the wet surface temperature is constant in time and space under a constant $Q_{n}$. Does it make any sense then to talk about a complementary relationship between water-limited $\left(E_{a}\right)$, and non-limited evaporation rates if the latter is a function of the distance along the wet surface? The answer is yes, provided the extent, $x_{f}$, of the wet surface is defined and a mean flux rate over that extent is obtained. 
Table 1

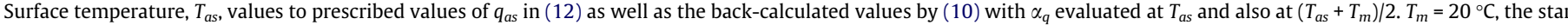
denotes the saturation level of $q$.

\begin{tabular}{|c|c|c|c|}
\hline$q_{a s}=c q^{*}\left(T_{m}\right), T_{m}=20^{\circ} \mathrm{C}, c$ & $T_{a s}\left[{ }^{\circ} \mathrm{C}\right]$ from $(12)$ & $T_{s}\left[{ }^{\circ} \mathrm{C}\right]$ from $(10)$ with $\alpha_{q}\left(T_{a s}\right)$ & $T_{s}\left[{ }^{\circ} \mathrm{C}\right]$ from $(10)$ with $\alpha_{q}\left[\left(T_{a s}+T_{m}\right) / 2\right]$ \\
\hline 0.95 & 21.75 & 20.06 & 20 \\
\hline 0.9 & 23.5 & 20.22 & 19.99 \\
\hline 0.85 & 25.26 & 20.5 & 19.99 \\
\hline 0.8 & 27 & 20.86 & 19.98 \\
\hline 0.7 & 30.51 & 21.88 & 19.93 \\
\hline 0.5 & 37.52 & 24.85 & 19.64 \\
\hline
\end{tabular}
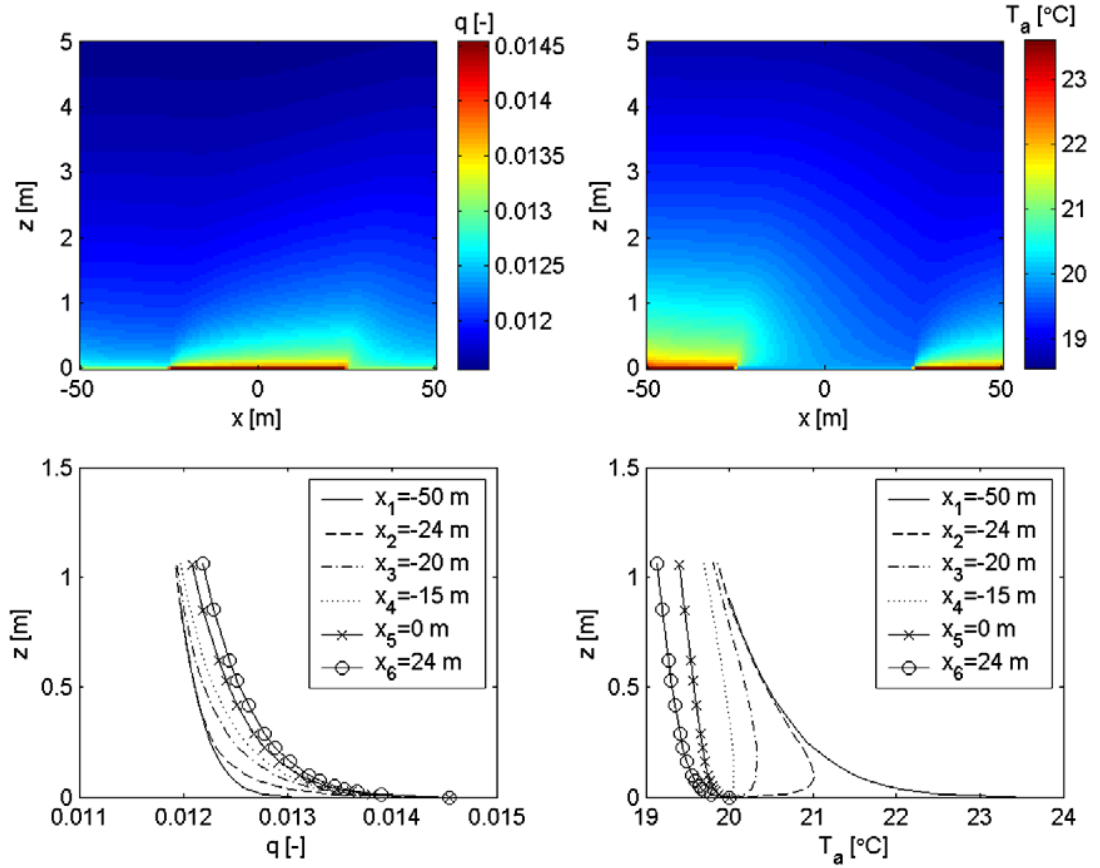

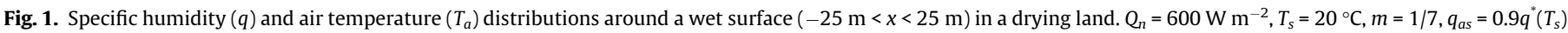
$z_{0}=0.01 \mathrm{~m}$, and $u_{*}=0.5 \mathrm{~ms}^{-1}$

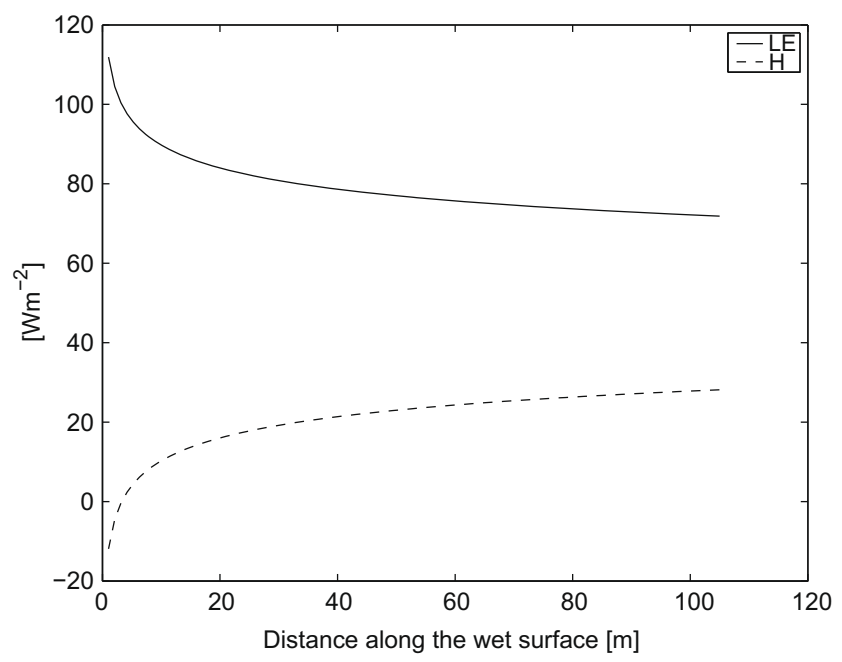

Fig. 2. Sensible $(H)$ and latent heat $(L E)$ fluxes along the wet surface under a constant $Q_{n}$ (and surface temperature). $Q_{n}=100 \mathrm{~W} \mathrm{~m}^{-2}, T_{s}=20^{\circ} \mathrm{C}, m=1 / 7$, $q_{a s}=0.8 q^{*}\left(T_{s}\right), z_{0}=0.0002 \mathrm{~m}$, and $u_{*}=0.24 \mathrm{~ms}^{-1}$.

\section{The complementary relationship}

Integrating ( 8 ) and (9) along $x$ between zero and $x_{f}$, and dividing by $x_{f}$ yields the mean fluxes as

$$
\begin{aligned}
& \bar{E}=E_{a}+c_{p} \rho b\left(\frac{a}{b x_{f}}\right)^{v} \frac{(1-v)^{2 v-2}(m+1)^{1-2 v}\left(q_{a s}^{*}-q_{a s}\right)}{\Gamma(v)\left(c_{p}+\alpha_{q} L_{e}\right)} \\
& \bar{H}=H_{a}-c_{p} \rho b\left(\frac{a}{b x_{f}}\right)^{v} \frac{(1-v)^{2 v-2}(m+1)^{1-2 v}\left(q_{a s}^{*}-q_{a s}\right) L_{e}}{\Gamma(v)\left(c_{p}+\alpha_{q} L_{e}\right)}
\end{aligned}
$$

where (13) is the so-called Sutton solution (1934), and $\bar{E}$ defines the potential evaporation rate, $E_{p}$, in (1). Before the CR can be investigated the fluxes over the originally uniformly wet and the ensuing drying surface are needed too. For the fully saturated initial conditions the Bowen ratio becomes $H / L E=\gamma / \delta\left(T_{\mathrm{z}=0}\right)$ where $\gamma$ is the psychrometric constant $\left(=c_{p} p / 0.622 \mathrm{~L}_{e} \approx 0.67 \mathrm{hPa} \mathrm{K}^{-1}\right.$ at $\left.20^{\circ} \mathrm{C}\right)$ and $\delta$ is the slope of the saturation vapor pressure curve at the surface temperature. Coupled with the constraint $L E+H=Q_{n}$, the initial fluxes can be obtained. This initial latent heat flux, $L E$, divided by the latent heat of vaporization, corresponds to $E_{w}$ in (1). In practice $E_{w}$ is most often defined by the Priestley-Taylor (1972) equation $E_{w}=$ $\alpha \delta(\delta+\gamma)^{-1} Q_{n}$, where $\alpha$ is the Priestly-Taylor parameter, meant to account for regional-scale advection of energy, and $\delta$ now is evaluated at the air temperature at $2 \mathrm{~m}$. Since such advection now is absent in the present model setup, this parameter has a value of unity. This way the difference in the wet environment evaporation rates, whether the latter are derived by the Bowen ratio or the Priestley-Taylor equation, is less than $1 \%$ (wet surface temperature of $20^{\circ} \mathrm{C}, Q_{n}=100 \mathrm{~W} \mathrm{~m}^{-2}$ ).

The drying surface equilibrium fluxes subsequently are derived first by prescribing either $q_{a s}$ or $T_{a s}$ in (12) to calculate the other, and then by integrating (3) for the equilibrium profiles of $\bar{q}$ and $\bar{T}$ 


$$
\begin{aligned}
& \bar{T}(z)=T_{a s}-\frac{H}{c_{p} b \rho(1-n)} z^{1-n} \\
& \bar{q}(z)=q_{a s}-\frac{E}{b \rho(1-n)} z^{1-n}
\end{aligned}
$$

with the assumption that above a certain elevation from the ground the profiles are only negligibly affected by the surface drying and the air remains close to saturation.

Table 2 lists the wet surface extent, $x_{f}$, required for a symmetric CR for different values of the air stability parameter, $m$, with surface $\left(z_{0}\right)$ and turbulence $\left(u_{*}\right)$ parameters representing a large open water body of the Finger Lakes region in upstate New York in the summer (Yeh and Brutsaert, 1971), as well as with a $z_{0}$ value characteristic of mixed vegetation of trees, bushes and grass (Brutsaert, 1982, p. 114). As expected, the wet surface extent increases with a weakening of the atmospheric stability, as the moisture is being transferred more effectively from the land into the upper regions of the air column, thus needing a longer fetch for the air to become close to saturation over the wet surface. Note that the extent of the wet surface needed for a symmetric CR is typically in the order of a $100 \mathrm{~m}$ under near-neutral atmospheric stability conditions for a smooth surface.

Eqs. (8), (9) and Fig. 2 demonstrate that both, the evaporation and the sensible heat flux, vary along the wet surface, while Table 2 also displays the required, certainly not negligible, extent of the wet surface for a symmetric CR between drying land evaporation and the mean evaporation rate of the wet surface. This contradicts the conclusion of Morton (1983) and Szilagyi (2001b) that the evaporation rate of a hypothetical evaporimeter (i.e., an almost zero-extent wet surface having insulated sides and bottom to prevent heat conduction across them) should yield a symmetric CR. In Morton's (1983) derivation a symmetric CR results only if one assumes that the temporal change in the drying land's sensible heat

Table 2

The $x_{f}$ values $[\mathrm{m}]$ as a function of $m$, required for a symmetric complementary relationship. (a) $z_{0}=0.0002 \mathrm{~m}, u_{*}=0.24 \mathrm{~ms}^{-1}$ (from Yeh and Brutsaert, 1971) and (b) $z_{0}=0.2 \mathrm{~m}$, and $u_{*}=0.24 \mathrm{~ms}^{-1}$

\begin{tabular}{llr}
\hline$m[-]$ & $(\mathrm{a})$ & $(\mathrm{b})$ \\
\hline $1 / 8$ & 40 & 7 \\
$1 / 7$ & 105 & 15 \\
$1 / 6$ & 312 & 31 \\
$1 / 5$ & 1150 & 72 \\
\hline
\end{tabular}

flux, $\Delta H$, equals the same change in the latent heat flux, $\Delta L E$, of the vanishing-size wet surface. As (8) and Fig. 2 indicate this is true in one point (somewhere in the middle) of the wet surface only, while it is also true for the mean $L E$ value (since $x_{f}$ was deliberately chosen so), neither representing a vanishing-size wet area. Szilagyi (2001b) attempted to demonstrate that the prescribed equality of the flux changes (i.e., $\Delta H=\Delta L E$ ) in Morton's derivation of a symmetric CR is not necessary over a homogeneous land area. The problem with the result of Szilagyi (2001b) is that it yields a symmetric CR the same as it does an asymmetric one by simply changing the lower reference level, required in his derivation, accordingly.

In practice the wet-surface or open-water evaporation rate is estimated by the Penman (1948) equation

$E_{P M}=\frac{\delta}{\delta+\gamma} Q_{n}+\frac{\gamma}{\delta+\gamma} f(u)\left(e_{a}^{*}-e_{a}\right)$

where the wind function, $f(u)$, is traditionally written as $f(u)=0.26\left(1+0.54 u_{2}\right)$ with $u_{2}\left[\mathrm{~ms}^{-1}\right]$ being the mean wind at $2 \mathrm{~m}$ above the ground. $e_{a}[\mathrm{hPa}]$ is the vapor pressure at the air temperature from the same elevation (both, $u$ and $e_{a}$, obtained upwind of the wet surface), the starred value denotes the saturation level, and the slope of the saturation vapor pressure curve, $\delta$, is also taken at the same air temperature. $Q_{n}$ must be in water depth equivalent of $\mathrm{mm} \mathrm{d}^{-1}$ for obtaining the evaporation rate in the same dimension. Obviously, (16) does not include the extent of the wet surface. However, it has been known that a symmetric CR employing (16) for $E_{p}$ in (1) yields realistic estimates of the actual evaporation rates (Brutsaert and Stricker, 1979; Hobbins et al., 2001a; Szilagyi and Jozsa, 2008). If so, then (16) can be expected to approximate the wet-surface/open-water evaporation rate of an area that has the proper extent to ensure a symmetric CR. Indeed, as Table 3 demonstrates, the $E_{P M}$ estimates are very close (i.e., within $10 \%$ ) to the analytical solution values of (13) for a smooth land surface (the small roughness value is representative of an open water body) under near-neutral atmospheric stability conditions. Interestingly, the Penman equation yields fairly good estimates (with a mean accuracy of about $15 \%$ ) of the mean evaporation rate of the smooth wet area with the above specified fetch values not just for a similarly smooth land surface but also for a much rougher one with a $z_{0}$ value two orders of magnitude larger, representing a realistic land surface upwind of a smooth open water area. Note that by changing the $z_{0}$ value, the Penman equation estimates are also af-

Table 3

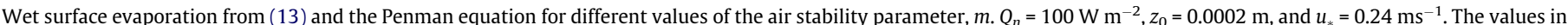

\begin{tabular}{|c|c|c|c|c|c|c|c|}
\hline \multirow[t]{2}{*}{$q_{\text {as }}=c q^{*}\left(T_{m}\right), T_{m}=20^{\circ} \mathrm{C}, c$} & \multirow[t]{2}{*}{$T_{\text {as }}\left[{ }^{\circ} \mathrm{C}\right]$ from $(12)$} & \multicolumn{3}{|c|}{$L E$ from $(13)\left[\mathrm{W} \mathrm{m}^{-2}\right], m$} & \multicolumn{3}{|c|}{$L E$ from Penman $\left[\mathrm{W} \mathrm{m}^{-2}\right], m$} \\
\hline & & $1 / 6$ & $1 / 7$ & $1 / 8$ & $1 / 6$ & $1 / 7$ & $1 / 8$ \\
\hline 1 & 20 & 68 & 68 & 68 & $68(68)$ & $67(68)$ & $67(68)$ \\
\hline 0.95 & 21.75 & 80 & 82 & 84 & $84(76)$ & $82(76)$ & $80(75)$ \\
\hline 0.9 & 23.5 & 92 & 96 & 99 & $100(85)$ & $96(84)$ & $92(83)$ \\
\hline 0.85 & 25.26 & 104 & 111 & 115 & $116(93)$ & $109(91)$ & $104(90)$ \\
\hline 0.8 & 27 & 117 & 125 & 131 & $131(101)$ & 122 (99) & 116 (97) \\
\hline
\end{tabular}
parentheses for the Penman equation estimates result from $z_{0}=0.2 \mathrm{~m}$, representing a more realistic rough land surface upwind to a smooth open water surface.

Table 4

\begin{tabular}{|c|c|c|c|c|}
\hline \multirow[t]{2}{*}{$q_{a s}=c q^{*}\left(T_{m}\right), T_{m}=20^{\circ} \mathrm{C}, c$} & \multirow[t]{2}{*}{$T_{a s}\left[{ }^{\circ} \mathrm{C}\right]$ from $(12)$} & \multicolumn{3}{|c|}{$L E$ from $(13)\left[\mathrm{W} \mathrm{m}^{-2}\right], x_{f}=105 \mathrm{~m}$} \\
\hline & & $x_{f} / 2$ & $x_{f}$ & $2 x_{f}$ \\
\hline 0.95 & 21.75 & 85 & 82 & 80 \\
\hline 0.9 & 23.5 & 101 & 96 & 92 \\
\hline 0.85 & 25.26 & 117 & 111 & 104 \\
\hline 0.8 & 27 & 134 & 125 & 116 \\
\hline
\end{tabular}

Sensitivity of the mean evaporation rate to changes in $x_{f}$ required for a symmetric complementary relationship for $m=1 / 7$. 
Table 5

The sensitivity of $\eta$ to changes in $x_{f}$ required for the validity of (1). $Q_{n}=100 \mathrm{~W} \mathrm{~m}^{-2}$, $T_{s}=20^{\circ} \mathrm{C}, m=1 / 7, q_{a s}=0.8 q^{*}\left(T_{s}\right), z_{0}=0.0002 \mathrm{~m}$, and $u_{*}=0.24 \mathrm{~ms}^{-1}$.

\begin{tabular}{|c|c|c|c|c|c|c|c|c|c|}
\hline$x_{f}(\mathrm{~m})$ & 5 & 10 & 25 & 50 & 105 & 200 & 500 & 1000 & 2000 \\
\hline$\eta$ & 2.67 & 2.49 & 2.29 & 2.14 & 2 & 1.89 & 1.74 & 1.63 & 1.5 \\
\hline
\end{tabular}

fected through a corresponding change in the wind velocity and diffusivity (see their definitions), as well as the temperature and moisture profiles [see (15)], even though the same wind function, specified by Penman, was employed throughout. In such a more realistic situation (under the same stability conditions) the mean evaporation rate as well as the fetch required for a symmetric CR can, however, be expected to differ somewhat from a uniformly smooth surface case due to the ensuing changes in the wind and $K$ profiles over the land and the simultaneous profile changes across the moisture discontinuity caused by a sudden drop in the $z_{0}$ value. The current theory, requiring a uniform roughness height, cannot explain the consequences of such a change in surface roughness. Note that on a daily basis, working with daily mean values, the assumption of near-neutral stability conditions is well justified.

In Table 3 the fetch has systematically been chosen to result in a symmetric CR. Since the $E_{P M}$ values are typically close to the ensuing mean evaporation rates, not only can it be concluded based on the analytical solution of the coupled 2-D steady turbulent heat and vapor transport equations that a symmetric CR indeed exists under a constant $Q_{n}$, provided the fetch or extent of the wet surface is chosen as a function of the air stability parameter, but it can also be stated that there already exists a fairly robust estimating technique, the Penman equation, that yields estimates of the mean evaporation rate of such an open water or wet surface. This of course is not by chance since: (a) for the calibration of the wind function small reservoir and lake evaporation measurements have also been used by Penman beside the application of sunken evaporation pans and (b) under (at least) near-neutral conditions even a relatively large deviation from the required fetch (Table 4 ) results in only slightly changed mean evaporation rates due to the near flat section of the $L E$ curve in Fig. 2 after its initial sharp decline with distance from the moisture discontinuity. It is for the same reason that the value of $\eta$ in (1), required for a symmetric $C R$, changes sluggishly with the extent of the wet surface (Table 5).

\section{Summary and conclusions}

The existence of a symmetric complementary relationship (CR) between actual (from a uniform drying land) and potential (the mean evaporation rate of a wet area having an along-the-wind extent in the order of a $100 \mathrm{~m}$ for a smooth surface) evaporation rates under near-neutral atmospheric stability conditions has been demonstrated with the help of a well-known analytical solution (Laikhtman, 1964; Yeh and Brutsaert, 1971) of the coupled 2-D turbulent heat and vapor transport equations. The assumptions necessary for a symmetric CR are: (a) the energy available for latent and sensible heat fluxes at the surface is constant; (b) the surface roughness is uniform; (c) the equilibrium profiles of the mean horizontal wind and turbulent diffusion coefficients remain constant in time; and (d) the turbulent diffusion coefficient is the same for heat and water vapor.

Under near-neutral atmospheric and uniformly smooth surface conditions the Penman equation estimates the mean evaporation rate of a wet surface remarkably well, i.e., within $10 \%$ of the analytical values. When the Penman equation is applied with a wind profile representative over a rough surface, its accuracy (assuming the mean wet surface evaporation and the required fetch would not change significantly) drops only slightly (to $15 \%$ on average). At the same time the mean evaporation rate of the smooth wet surface changes only weakly with fetch around the value required for a symmetric CR. All this is in support of choosing the Penman equation as an effective practical tool for estimating the potential evaporation rate in (1) by Brutsaert and Stricker (1979) in their Advection-Aridity (AA) model, the first published evaporation estimation method that relies on a symmetric $C R$.

Although the requirement of a constant energy term, $Q_{n}$ (as well as unchanging atmospheric profiles of wind speed and diffusivity) for a symmetrical CR seems overly restrictive at the drying land surface, certain land-atmosphere radiation feedbacks may exist that can lead to such a near constant $Q_{n}$. Whether this truly happens or not in reality or to what degree, and how the violation of the other requirements above affects the CR in practice will be told by the future success or failure of the experimental as well as routine applications of the CR-based evaporation estimation methods.

\section{Acknowledgment}

This work has partially been supported by the European Union's Climate Change and Variability: Impact on Central and Eastern Europe (CLAVIER) FP6 project.

\section{References}

Bouchet, R.J., 1963. Evapotranspiration reelle, evapotranspiration potentielle, et production agricole. Ann. Agron. 14, 543-824.

Brutsaert, W., 1982. Evaporation into the Atmosphere. D. Reidel, Dordrecht. p. 299

Brutsaert, W., Stricker, H., 1979. An Advection-Aridity approach to estimate actual regional evapotranspiration. Water Resour. Res. 15, 443-449.

Brutsaert, W., Parlange, M.B., 1998. Hydrologic cycle explains the evaporation paradox. Nature 396, 30.

Crago, R., Crowley, R., 2005. Complementary relationships for near-instantaneous evaporation. J. Hydrol. 300, 199-211.

Hobbins, M.T., Ramirez, J.A., Brown, T.C., Claessens, L.H.J.M., 2001a. The complementary relationship in estimation of regional evapotranspiration: the complementary relationship areal evaporation and Advection-Aridity models. Water Resour. Res. 37 (5), 1367-1387.

Hobbins, M.T., Ramirez, J.A., Brown, T.C., 2001b. The complementary relationship in estimation of regional evapotranspiration: an enhanced Advection-Aridity model. Water Resour. Res. 37 (5), 1389-1403.

Kahler, D.M., Brutsaert, W., 2006. Complementary relationship between daily evaporation in the environment and pan evaporation. Water Resour. Res. 42, W05413. doi:10.1029/2005WR004541.

Kim, C.P., Entekhabi, D., 1997. Examination of two methods for estimating regional evaporation using a coupled mixed layer and land surface model. Water Resour Res. 33 (9), 2109-2116.

Laikhtman, D.L., 1964. Physics of the Boundary Layer of the Atmosphere. Sivan Press, Israel.

LeDrew, E.F., 1979. A diagnostic examination of a complementary relationship between actual and potential evapotranspiration. J. Appl. Meteorol. 18, 495501.

Lhomme, J.P., 1997a. A theoretical basis for the Priestley-Taylor coefficient. Bound Layer Meteorol. 82, 179-191.

Lhomme, J.P., 1997b. An examination of the Priestley-Taylor equation using a convective boundary-layer model. Water Resour. Res. 33, 2571-2578.

Lhomme, J.P., Guilioni, P., 2006. Comments on some articles about the complementary relationship. J. Hydrol. 323, 1-3.

McNaughton, K.G., Spriggs, T.W., 1989. An evaluation of the Priestley-Taylor equation and the complementary relation using results from a mixed layer model of the convective boundary layer. In: Black, A.T., Spittlehouse, D.L. Novak, M.D., Price, D.T. (Eds.), Estimation of Areal Evapotranspiration, IAHS Press, Wallingford, United Kingdom.

Monteith, J.L., 1973. Principles of Environmental Physics. Elsevier. p. 241.

Morton, F.I., 1983. Operational estimates of areal evapotranspiration and their significance to the science and practice of hydrology. J. Hydrol. 66, 1-76.

Penman, H.L., 1948. Natural evaporation from open water, bare soil, and grass. Proc Roy. Soc. London A193, 120-146.

Pettijohn, J.C., Salvucci, G.D., 2006. Impact of an unstressed canopy conductance on the Bouchet-Morton complementary relationship. Water Resour. Res. 42 W09418, doi:10.1029/2005WR004385.

Priestley, C.H.B., Taylor, R.J., 1972. On the assessment of surface heat flux and evaporation using large-scale parameters. Mon. Weather Rev. 100, 81-92.

Ramirez, J.A., Hobbins, M.T., Brown, T.C., 2005. Observational evidence of the complementary relationship in regional evaporation lends strong support for Bouchet's hypothesis. Geophys. Res. Lett. 32, L15401. doi:10.1029/ 2005GL023549. 
Sugita, M., Usui, J., Tamagawa, I., Kaihotsu, I., 2001. Complementary relationship with a convective boundary layer model to estimate regional evaporation. Water Resour. Res. 37 (2), 353-365.

Sutton, O.G., 1934. Wind structure and evaporation in a turbulent atmosphere. Proc. Roy. Soc. London A146, 701-722.

Szilagyi, J., 2001a. Modeled areal evaporation trends over the conterminous United States. J. Irrig. Drainage Eng. 127 (4), 196-200.

Szilagyi, J., 2001b. On Bouchet's complementary hypothesis. J. Hydrol. 246, 155158.

Szilagyi, J., Katul, G.G., Parlange, M.B., 2001. Evapotranspiration intensifies over the conterminous United States. J. Water Resour. Plan. Manag. 127 (6), 354-362.
Szilagyi, J., 2007. On the inherent asymmetric nature of the complementary relationship of evaporation. Geophys. Res. Lett. 34, L02405. doi:10.1029/ 2006 GL028708.

Szilagyi, J., Jozsa, J., 2008. New findings about the complementary relationshipbased evaporation estimation methods. J. Hydrol. 354, 171-186.

Szilagyi, J., Jozsa, J., 2009. An evaporation estimation method based on the coupled $2-D$ turbulent heat and vapor transport equations. J. Geophys. Res. 114, D06101. doi:10.1029/2008JD010772.

Yeh, G.-T., Brutsaert, W., 1971. A solution for simultaneous turbulent heat and vapor transfer between a water surface and the atmosphere. Bound. Layer Meteor. 2, 64-82. 